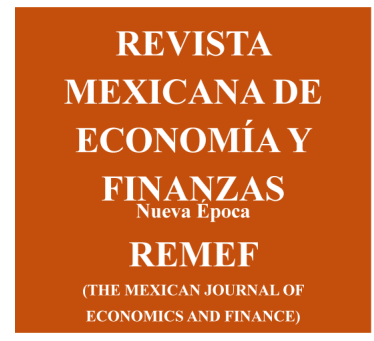

\title{
Revista Mexicana de Economía y Finanzas Nueva Época
}

Volumen 15 Número 3, Julio - Septiembre 2020, pp.295-311

DOI: https://doi.org/10.21919/remef.v15i3.514

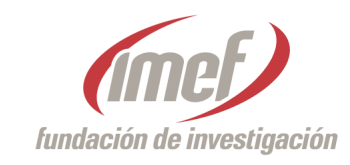

(Recibido: 31/marzo/2020, aceptado: 29/junio/2020)

\section{Tasa de política monetaria en México ante los efectos de Covid-19}

\author{
Armando Sánchez Vargas ${ }^{1}$ \\ Universidad Nacional Autónoma de México, México \\ Francisco López-Herrera ${ }^{2}$ \\ Universidad Nacional Autónoma de México, México
}

\section{Resumen}

La crisis económica alimentada por el Covid-19 ha producido choques de oferta y demanda que afectan al producto potencial y a las preferencias de los consumidores, impacto que sugiere que la tasa real neutral de interés se modificó y, por ende, la tasa real de interés debe ajustarse para no generar una contracción económica más profunda en México. Estimamos el valor de la tasa neutral utilizando el método de Laubach y Williams, basado en el filtro de Kalman, y confirmamos los resultados con un modelo cointegrado de vectores autorregresivos (CVAR). Los resultados muestran que la tasa real neutral, teóricamente congruente con el pleno empleo y la meta de inflación, se reduce hasta $0.1 \%$ y la nominal hasta $3.1 \%$. La originalidad se debe la determinación empírica de la tasa neutral con base en dos métodos distintos para México y su utilización para aproximar que tan expansiva debe ser la política monetaria en México en la etapa de la COVID. Los resultados sugieren la existencia de suficiente espacio para una política monetaria expansiva que permita enfrentar la crisis e impulsar la actividad económica durante al menos tres años.

Clasificación JEL: E43, E52, E58, E66, F62.

Palabras claves: Covid-19, pandemia, política monetaria, tasa de interés, crisis mundial.

\section{Monetary policy rate in Mexico due to the effects of Covid-19}

\section{Abstract}

The economic crisis feeded by Covid-19 has produced supply and demand shocks that affect the potential product and consumer preferences, an impact that suggests that the neutral real interest rate was modified and, therefore, the real interest rate should be adjusted so as not to generate a deeper economic contraction in Mexico. We estimate the value of the neutral rate using the Laubach and Williams method, based on the Kalman filter, and confirm the results with a cointegrated vector of autoregressions (CVAR) model. The results show that the neutral real rate, theoretically consistent with the full employment rate and the inflation target in Mexico, is reduced to $0.1 \%$ and the nominal to $3.1 \%$. The empirical determination of the neutral rate based on two different methods for Mexico and its use to approximate how expansive the monetary policy should be in Mexico in the COVID stage. The results suggests the existence of enough room for an expansionary monetary policy that allows facing the crisis and boost economic activity for at least three years.

JEL Classification: E43, E52, E58, E66, F62.

Keywords: Covid-19, pandemic, monetary policy, interest rate, global crisis.

\footnotetext{
${ }^{1}$ Instituto de Investigaciones Económicas. Circuito Mario de la Cueva s/n, Ciudad de la Investigación en Humanidades,

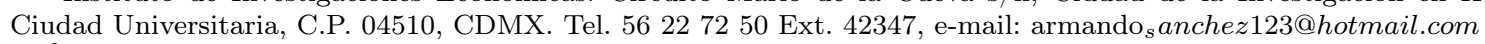

${ }^{2}$ División de Investigación, Facultad de Contaduría y Administración. Circuito Exterior s/n, Ciudad Universitaria, Delegación Coyoacán, C.P. 04510, México, Ciudad de México. Tel. 562284 94, e-mail: francisco_lopez_herrera@yahoo.com.mx
} 


\section{Introducción}

¿Cuál debería ser la postura de política monetaria del Banco de México (Banxico) durante y después la contracción económica generada por las medidas de sanidad para enfrentar la pandemia de Covid-19? Para incentivar el consumo y la inversión Banxico podría bajar el nivel de su tasa de política monetaria como complemento al aumento de la liquidez que ha instrumentado en el sistema financiero para estimular la economía mediante el canal de crédito. Para ello es importante conocer con la mayor precisión posible cuánto Banxico podría reducir dicha tasa sin afectar negativamente la dinámica inflacionaria.

La decisión del nivel óptimo de la tasa de referencia podría elegirse de manera muy sencilla si el Banco Central conociera el valor de la tasa real neutral $(r *)$, aquella que es compatible con el PIB potencial $\left(\mathrm{y}^{*}\right)$ y en la que la inflación converge a su nivel objetivo (Laubach y Williams, 2001; Taylor, 1993). Sin embargo, dicho valor no es observable y puede cambiar con el tiempo (Clarida, 2015), por lo que en el presente artículo analizamos cuáles deberían ser, aproximadamente, los valores de la tasa de interés real y nominal para México con una perspectiva de mediano plazo. Para ello, estimamos la tasa de interés real neutral no observable que prevalecería en el futuro cercano hasta que la economía se haya recuperado de las fluctuaciones económicas generadas por la crisis y el PIB crezca a su tasa potencial, ya que también se requiere saber en qué momento se podría retirar el estímulo monetario.

Después de esta sección, el documento sigue la estructura enunciada a continuación. En la segunda sección se señalan las principales perspectivas económicas originadas a partir del brote mundial de Covid-19. La tercera sección ofrece una revisión de literatura teórica y empírica sobre la determinación de la tasa de interés neutral. En la cuarta sección se analizan hechos estilizados sobre la tasa de interés comparando su dinámica durante las crisis de fines de la década pasada y la actual. La quinta sección describe la metodología usada en las estimaciones cuyos resultados se presentan y discuten en la sexta sección. Finalmente, se enuncian las conclusiones.

\section{Perspectivas económicas originadas por Covid-19}

Como lo señalan Ozili y Arun (2020), debido a las presiones derivadas de la guerra comercial entre China y Estados Unidos, las elecciones presidenciales en este último país y el Brexit, las predicciones económicas en 2019 eran de crecimiento moderado de la economía mundial para 2020, por ejemplo, el FMI lo proyectaba en $3.4 \%$. Sin embargo, el brote del Covid-19 contribuyó a modificar esas perspectivas, ${ }^{3}$ dando lugar a temores inducidos por la incertidumbre sobre los efectos de esa enfermedad en las economías del mundo; las estimaciones de Barro et al. (2020) sugieren caídas de $6 \%$ para el PIB y de $8 \%$ para el consumo privado en un país típicamente afectado por la crisis.

Las consecuencias económicas inmediatas de la pandemia de Covid-19 en los países afectados por ella ya están a la vista, Nicola et al. (2020) destacan la pérdida de empleos, al igual que la caída en la demanda de manufacturas y otras mercancías, en tanto que ha crecido la demanda de suministros médicos. Las expectativas sobre los impactos de corto plazo se deterioraron rápidamente, como lo muestra para el caso de Estados Unidos el análisis de Dietrich et al. (2020) en el que documentan que, en las tres semanas inmediatas a partir del 10 de marzo de 2020, la caída esperada en el producto incrementó 15

Según Gopinath (2020), durante la epidemia se han presentado choques del lado de la oferta debido a que la interrupción de las actividades de las empresas ha disminuido el nivel de producción, así como choques por la caída de la demanda debido a la renuencia a gastar por parte de los consumidores y empresas. La caída

\footnotetext{
${ }^{3}$ En la formación de expectativas negativas para el desempeño de este año también juega un papel importante la guerra de los precios del petróleo, cuyos efectos negativos para los países productores fueron ampliados por la irrupción de la enfermedad.
} 
del consumo en los países más desarrollados implicó para América Latina una fuerte caída en los precios de sus productos de exportación, produciendo pérdidas en las paridades cambiarias y efectos recesivos dada la sensibilidad de la economía a esos precios como lo señalan Schmitt-Grohé y Uribe (2018).

Evidentemente, todos los países del mundo han enfrentado un dilema muy complejo al tener que optar por las estrategias para contener la expansión de la enfermedad general, tratando al mismo tiempo que los daños a la economía sean los menores posibles, es decir, como resolver un problema económico local inducido por la administración de un problema de salud mundial. Con base en su análisis sobre la interacción entre las decisiones de los agentes económicos y la severidad de la epidemia, Eichenbaum et al. (2020) plantean que las decisiones de aislamiento social reducen los efectos de la pandemia, pero incrementan la intensidad de la recesión. De acuerdo con su modelo, los efectos de la política óptima de contención en Estados Unidos sobre la oferta y demanda agregadas inducen una recesión larga y persistente, sin embargo, permite salvar aproximadamente medio millón de vidas. Al respecto, Barro et al. (2020) consideran que, en todo caso, la enorme pérdida potencial de vidas justifica el gasto sustancial de recursos para intentar limitar el daño.

Las consecuencias para las economías del mundo en el largo plazo, como lo señala Zhang et al. (2020), son menos claras. Gourinchas (2020) reconoce que la contención de la pandemia es la mayor prioridad y que es razonable suponer que se regrese a un nivel de actividad semejante al que se observaba antes de la crisis en lugar de uno más alto, sin embargo, podría haber un incremento notable posteriormente debido al gasto en bienes durables pospuesto con motivo de la crisis. A pesar de ello, otro reto de la problemática es que la profundidad y duración de la crisis económica post-pandemia no se puede aún determinar con exactitud dado que hay diversos factores que considerar. Algunos economistas sugieren considerar que Cerra y Saxena (2017) muestran evidencia sobre los efectos negativos permanentes que pueden tener las crisis y recesiones sobre el producto; en el caso de Estados Unidos el producto no regresa a la tendencia previa, a pesar de que se observen posteriormente al episodio crítico reducción de la tasa de desempleo acompañado, lógicamente, de mayor empleo de la capacidad instalada.

Furman (2020), Odendahl y Springford (2020) y Galí (2020) señalan diversos riesgos posibles, algunos de tipo financiero como las quiebras empresariales, falta de liquidez e insolvencia de los bancos, otro dado por la probabilidad de colapsos en la actividad de industrias y ramas económicas, además de las posibilidades de que el espacio fiscal y el nivel de ingresos de algunos países pudieran ser insuficientes ante los costos para mitigar la pandemia. Es conveniente indicar que Bell y Lewis (2004) habían ya advertido que un problema del choque que puede ocurrir en la economía como consecuencia de una epidemia es el direccionamiento del ahorro de las familias hacia el tratamiento de la enfermedad, en lugar de ir hacia los diferentes tipos de inversión, condición agravada por la reducción del ahorro debido a las pérdidas en el ingreso; produciendo en última instancia una caída en el nivel del capital físico. Bell y Lewis (2004) consideran que determinar las consecuencias de una epidemia para la acumulación capital humano es un asunto complejo, pero señalan que en última instancia el efecto es una reducción de la calidad de la fuerza laboral más que de su cantidad, pues dependen del grupo de edades más afectadas por la pandemia.

En su estudio sobre 11,943 empresas con matrices en 84 país, Hassan et al. (2020) encuentran que en la medida en que la Covid-19 se extendió a escala mundial durante el primer trimestre de 2020, las principales preocupaciones en las empresas se relacionaron con el colapso de la demanda, el aumento en la incertidumbre y la ruptura de las cadenas de aprovisionamiento mas, curiosamente, no con el financiamiento, el que parecía ser en última instancia un asunto que les parecía muy poco preocupante. Sin dejar de reconocer las características específicas de la situación actual, esos estudiosos señalan que esta pandemia es un caso extremo, pero que se puede aprender mucho sobre la capacidad adaptativa del sector corporativo a partir de lo ocurrido en otros precedentes inmediatos, como SARS y H1N1, y ofrecen evidencia de que las empresas que tuvieron experiencias en eventos como esos tienen expectativas más positivas sobre su capacidad para enfrentar el 
brote de la nueva enfermedad. También observan en sus resultados que hay empresas que ven con optimismo en la situación actual pues preveen oportunidades en los nuevos mercados que aparecen o aparecerán durante las secuelas o, incluso, algunas oportunidades se avizoran en los mercados cuyo funcionamiento regular se ha visto trastornado.

El debate sobre la idoneidad de la respuesta de los diferentes países del mundo para superar la crisis postpandemia y abrir paso a la recuperación económica es amplio. Gopinath (2020) considera que se requiere instrumentar medidas fiscales y monetarias, incluyendo medidas que involucran a los mercados financieros, para auxiliar a las familias y empresas mediante transferencias de efectivo, subsidios al salario y alivio fiscal. Un tanto escéptico sobre la eficacia de la llamada política monetaria no convencional en el largo plazo e indicando las limitaciones de los estímulos fiscales para tener efectos inmediatos, para el caso estadounidense Krugman (2020) propone un incremento de $2 \%$ en la inversión pública y que se mantenga ese nivel aumentado en forma permanente. Cochrane (2020) considera que las bajas en las tasas de interés al inicio de la crisis, respuesta que han tenido muchos países ante la problemática, resulta conveniente en la medida que proporciona liquidez a la economía, sin embargo, duda que sean suficientes para resolver el problema de largo plazo en Estados Unidos, donde las tasas de interés han bajado a niveles cercanos a cero, naturalmente una condición muy distinta al caso de México en el cual la tasa de interés parecer estar lejos aún del cero.

El debate abre la puerta a considerar el margen de maniobra para instrumentar políticas adecuadas para superar los estragos económicos de la pandemia y recuperar la marcha económica. En particular, dado el énfasis que la política monetaria mexicana debe tener para mantener la inflación en un nivel bajo, es pertinente analizar si su instrumento para tales efectos, la tasa de interés, puede reducirse en la coyuntura actual, sin perder de vista el mandato de una inflación baja.

\section{La tasa de interés neutral: teoría y evidencia empírica}

En ausencia de choques de oferta y demanda, la media aritmética de la tasa de interés real de política monetaria, en un horizonte temporal dado, podría ser un buen estimador para aproximar el valor de la tasa de interés real neutral. Sin embargo, dado que la economía es dinámica y pueden presentarse dichos choques de oferta y demanda que generan fluctuaciones bruscas, la media aritmética de $r$ es un predictor sesgado de la tasa de interés real neutral $(r *)$ (Laubach y Williams, 2015).

La estimación de la tasa neutral es problemática dado que depende de parámetros que cambian con el tiempo. Además, esta tasa también cambiará en el caso de una crisis como la generada por el Covid-19 pues habrá cambios súbitos de los parámetros y con mayor intensidad que en una situación de normalidad. En una sección posterior estimamos la tasa real neutral para la economía mexicana utilizando el modelo aquí descrito, lo que nos permitirá discutir que tan expansiva debe ser la postura de Banxico ante la severa crisis económica. Esta crisis será de carácter temporal, pero generará choques continuos que se reflejan como una crisis sanitaria que, en el corto plazo, dará lugar a efectos recesivos de mediano plazo en la economía mexicana.

Varios esfuerzos se han realizado para estimar la tasa de interés real neutral de corto y largo plazos. Utilizando modelos con parámetros cambiantes en el tiempo, Méssonnier y Renne (2007) estiman una tasa de interés natural que varía en el tiempo (TVNRI) para la zona euro para el periodo comprendido entre 1979 y 2004. Estos autores hacen uso del modelo propuesto por Laubach y Williams (2003) y utilizan el filtro de Kalman (1960) para estimar un modelo del espacio de estados que incluye la curva de Phillips y una ecuación de demanda agregada. Los autores encuentran que la relación entre la TVNRI y las fluctuaciones de baja frecuencia del crecimiento potencial de la producción son fuertes. Los intervalos de confianza del filtro de Kalman resultan ser muy amplios y la percepción errónea de la tasa de interés natural también 
podría ser sustancial. Debido a la incertidumbre que señalan Laubach y Williams (2003), los autores no recomiendan hacer uso prescriptivo de las estimaciones de la tasa de interés natural. Juzgan conveniente evaluar la incertidumbre que rodea a las variables no observadas para hacer un estudio de la solidez de las reglas de política monetaria.

España-Arias (2008) estimó la tasa neutral de interés para la economía de Uruguay en el periodo 19922007, encontrando evidencia favorable a la metodología de Laubach y Williams (2003) y al uso del filtro de Kalman (1960), pero adoptó también la modificación de Mésonnier y Renne (2004) para tomar en cuenta las características de la economía uruguaya. Los resultados obtenidos mostraron que, hasta 2001, la tasa real de interés osciló alrededor del valor esperado de la tasa neutral en la economía uruguaya. Esto implica que la posición de política monetaria del banco central, en ese periodo, fue compatible con una tasa neutra real que se encuentra alrededor de la que es compatible con el pleno empleo de las capacidades productivas y el objetivo de inflación del banco central.

Goyal y Arora (2016) analizan la tasa de interés natural, la producción potencial y el crecimiento tendencial de la India. Los autores emplean el filtro de Kalman y un modelo semiestructural neokeynesiano de la demanda y oferta agregadas con expectativas adaptativas. Goyal y Arora encuentran que las perturbaciones de los precios de los alimentos reducen el nivel de consumo de subsistencia y aumentan la disposición a trabajar para protegerlo, de esta manera, se reduce la tasa de interés natural. Los resultados sugieren que las combinaciones de crecimiento e inflación se explican mejor por una oferta agregada plana sujeta a cambios frecuentes que por una oferta agregada más estable y pronunciada.

Fuentes y Gredig (2008) estimaron la tasa de interés neutral para Chile entre 2002 y 2007 usando un modelo semiestructural basado en la propuesta de Laubach y Williams y en datos macroeconómicos. Los supuestos fueron: 1) la economía puede ser modelada como una curva IS y una curva de Phillips (estas dos retrospectivas y con varianzas constantes), y 2) la tendencia estocástica sigue un proceso de paseo aleatorio y que el comportamiento del premio por plazo sigue un proceso $\mathrm{AR}(1)$. Los resultados mostraron que la tasa de interés neutral no es una variable constante en el tiempo y que está significativamente relacionada con el crecimiento potencial de la economía.

Behera et al. (2017) se apoyan en el modelo de crecimiento de Ramsey (1928), basado en la macrodinámica del nuevo keynesianismo y del filtro de Kalman para estimar la tasa de interés natural de India. Ellos encuentran una brecha de la tasa de interés real negativa, cuando la inflación fue alta. La tasa de interés neutral estimada en India es variable en el tiempo y las estimaciones son sensibles a la elección metodológica para desinflar la tasa de interés nominal. Encuentran que la tasa de interés natural estimada disminuye después de la crisis mundial y que la tasa de interés real permaneció negativa durante la década. Derivada de la inflación de dos dígitos que duró cinco años (2009-2014), la brecha de la tasa de interés real casi se cerró y se volvió marginalmente positiva en la segunda mitad de 2014-2015, esto reflejó el impacto combinado de una política monetaria que se centra en la inflación y que ayudó al impulso de la tasa de política real más cerca de la tasa natural estimada.

Para el caso de México, Carrillo et al. (2018) estiman la $r *$ de corto y largo plazos para México y Estados Unidos, apoyándose en la regla de Taylor, una adaptación del modelo de Laubach y Williams (2003), y un modelo BVAR. Los autores encontraron que los modelos sugieren una reducción de $r *$ tanto en el corto como en el largo plazo a raíz de la crisis financiera iniciada en Estados Unidos durante 2008, ampliamente conocida como la crisis subprime, y un crecimiento moderado desde 2014. Sin embargo, explican que la caída a corto plazo de $r *$ en México después de la crisis de 2008 se explica mejor por factores transitorios como las condiciones sostenidas de holgura económica en México y Estados Unidos. 


\section{Hechos estilizados: tasa neutral y las crisis de 2008 y 2020}

La figura 1 muestra la relación empírica entre la tasa de interés nominal (tasa de referencia del Banxico, TIIE a 1 día) y la inflación. Observamos que ambos indicadores presentan fluctuaciones a lo largo del tiempo. Específicamente, la crisis financiera de 2008 ocasionó un choque de liquidez que afectó a la demanda, lo cual repercutió en la tasa de interés de corto plazo, que pasó de $8.4 \%$ en 2008Q4 a 4.5\% en 2009Q4. Es decir, Banxico decidió reducir su tasa de interés con la finalidad de disminuir la caída de la actividad económica causada por la crisis financiera. La economía mexicana se contrajo a tal grado que la inflación pasó de $6.1 \%$ a $3.9 \%$ en el mismo lapso. Esto indica que los agentes económicos dejaron de gastar durante y después de la crisis económica.

En la última parte de la figura 1, se observa que el efecto del Covid-19 se sumó a la contracción económica que venía presentándose en México. Notamos que la tasa de interés nominal pasó de 8.3\% en 2019Q1 a 7.1\% en 2020Q1. Mientras que la inflación se mantiene en un nivel bajo. Por otro lado, la imagen 2 ofrece evidencia de que también la tasa de interés real se ha visto afectada hacia la baja. Banxico ha decidido comenzar a relajar su política monetaria dada la magnitud de la crisis que ya está presente en la actividad económica.

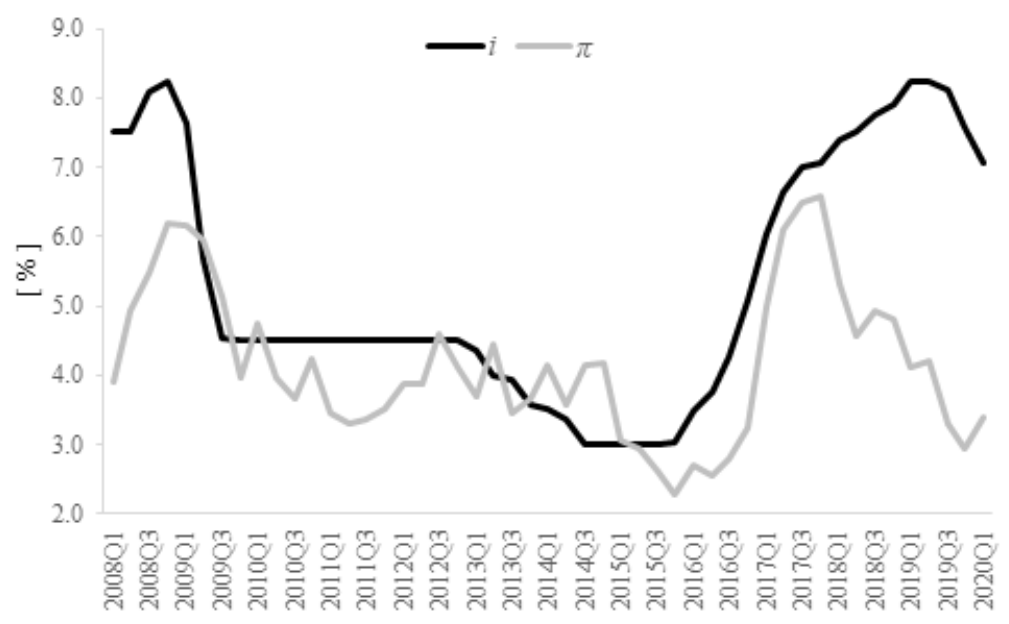

Figura 1. Tasa de interés nominal (i) e inflación $(\pi)$

Fuente: Elaboración propia con datos de Banxico (2019) e INEGI (2019).

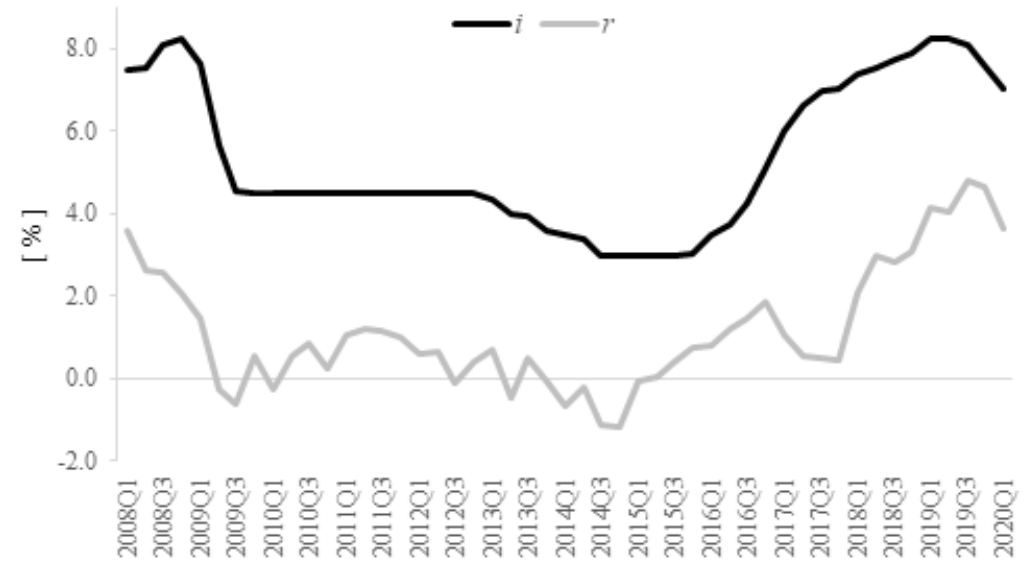

Figura 2. Tasa de interés nominal (i) y real (r)

Fuente: Elaboración propia con datos de Banxico (2019) e INEGI (2019). 
La figura 2 también confirma que la tasa de interés real redujo su valor debido a los efectos de la crisis de 2008, observamos que i bajó de manera significativa y después recuperó su valor previo a la crisis. La figura 2 también muestra que entre 2017 y 2018 las tasas de interés real y nominal alcanzan nuevamente su nivel observado previo a la crisis de 2008. Lo cual podría sugerir que después del choque del Covid-19, las tasas podrían regresar a su trayectoria en los trimestres posteriores a niveles similares a la crisis de 2008 donde la tasa nominal tuvo valores de alrededor de 4.5 por un tiempo prolongado debido a los efectos negativos de la crisis. Este hecho estilizado, sugiere que si la crisis del Covid-19 alcanza niveles de caída del producto similares a la crisis de 2008, entonces la tasa nominal de interés debería reducirse para alcanzar niveles de, al menos, de alrededor de un 4.5 por ciento y la tasa real de alrededor de 1 por ciento. Esto para conseguir que la recuperación económica sea más rápida y eficiente y con apoyo de una política monetaria expansiva suficiente.

\section{Aspectos metodológicos}

Laubach y Williams (2001, 2003) definieron un modelo macroeconómico multivariado para capturar el movimiento del producto y la inflación, permitiendo estimar el valor de la tasa de interés neutral que cambia con el tiempo. La estimación de este modelo está basada en el filtro de Kalman multivariado en el contexto de un modelo del espacio de los estados para obtener el valor de dicha tasa. De acuerdo con la teoría planteada en dicho modelo, la tasa de interés neutral no es fija en el tiempo y depende de los cambios en las preferencias y en la tasa de crecimiento del producto.

En principio, el modelo asume que el resultado del problema de optimización de la utilidad intertemporal de los consumidores muestra la relación existente entre la tasa de interés real y el crecimiento económico:

$$
r_{t}=\frac{1}{\sigma} g_{c}+\theta
$$

donde $\sigma$ es la elasticidad de substitución del consumo de los hogares, gc es la tasa de crecimiento del consumo per cápita y $\theta$ es la tasa de preferencias de los consumidores en el tiempo.

La teoría también sugiere que la tasa de interés neutral cambia si hay modificaciones en la tasa de crecimiento del producto o si hay cambios en las preferencias de los hogares debido a choques de demanda o a choques de política fiscal. Entonces, los fundamentos de la economía incidirán sobre el valor de $r_{t}$ y también

en el valor de $r *$. En función de ello, una crisis como la del Covid-19 que modifica de manera brusca los fundamentos de la economía podría ocasionar cambios en la tasa neutral real de interés. Así, es importante determinar el cambio en el valor de $r *$ con una metodología que permita que los parámetros cambien el tiempo.

En este contexto, Laubach y Williams (2003) y Holston et al. (2017) asumen que la relación entre la tasa neutral y la tasa de crecimiento del producto está gobernada por la siguiente ley de movimiento

$$
r_{t}^{*}=c g_{t}+z_{t}
$$

donde $g_{t}$ es la tasa de crecimiento tendencial, $z_{t}$ son otros determinantes de $r *$ que siguen un proceso autorregresivo.

Se asume que la variable $z_{t}$ de la ecuación (2) sigue un proceso autorregresivo:

$$
z_{t}=D_{z}(L) z_{t-1}+\varepsilon_{3, t}
$$

En su modelo Laubach y Williams (2003) también incluyen una ecuación en forma reducida de la ecuación 
IS:

$$
\widetilde{y}_{t}=a_{y, 1} \widetilde{y}_{t-1}+a_{y, 2} \widetilde{y}_{t-2}+\frac{a_{r}}{2} \sum_{j=1}^{2}\left(r_{t-j}-r_{t-j}^{*}\right)+\varepsilon_{1, t},
$$

donde $\widetilde{y}_{t}$ es la brecha del producto, $r_{t}$ es la tasa de interés real, $r_{t} *$ es la tasa natural o neutral de interés, la cual no es observable, y $\varepsilon_{, t}$ es el error serialmente no correlacionado. $\widetilde{y}_{t}=100 *\left(y t^{\iota} y t *\right)$ se obtiene como la resta de las variables en logaritmo del PIB real $\left(y_{t}\right)$ y el PIB potencial $\left(y_{t} *\right)$, esta última variable tampoco es observable. En la ecuación (4) $\widetilde{y}_{t}$ está determinado por sus rezagos, así como por una media móvil de la brecha de tasa de interés real; también se incluyen los choques transitorios y permanentes ya que ambos afectan a la brecha del producto. Los choques transitorios de corto plazo generan retrasos en la brecha del producto, contrario a lo que sucede con los choques permanentes que son derivados de los cambios en la tasa neutral $r *$. La ecuación (5) define la inflación en función de sus rezagos $B_{\pi}$, la brecha del producto $\left(\widetilde{y}_{t-1}\right)$, los precios relativos de las importaciones y del precio del $\operatorname{crudo}^{4}\left(\pi_{t}^{I}\right.$ y $\left.\pi_{t-1}^{O}\right)$, y un término de error $\left(\varepsilon_{2 t}\right)$ :

$$
\pi_{t}=B_{\pi}(L) \pi_{t-1}+b_{y} \widetilde{y}_{t-1}+b_{i}\left(\pi_{t}^{I}-\pi_{t-1}\right)+b_{o}\left(\pi_{t-1}^{O}-\pi_{t-1}\right)+\varepsilon_{2, t} .
$$

Según el modelo, los componentes de $y *$ y $g_{t}$ evolucionan como una caminata aleatoria:

$$
\begin{gathered}
y_{t}^{*}=y_{t-1}^{*}+g_{t-1}+\varepsilon_{4, t} \\
g_{t}=g_{t-1}+\varepsilon_{5, t},
\end{gathered}
$$

$\varepsilon_{3, t}, \varepsilon_{4, t}, \mathrm{y} \varepsilon_{5, t}$ son serial y contemporáneamente no correlacionadas.

De acuerdo con Laubach y Williams (2003), al aplicar el filtro de Kalman es probable que las desviaciones estándar del término de error de z $\left(\sigma_{3}\right)$ y de la tasa de crecimiento tendencial del PIB $\left(\sigma_{5}\right)$ estén sesgadas hacia cero, llevando al problema de pile-up (Stock, 1994). Para solucionarlo, Laubach y Williams (2003) emplean el estimador de media insesgada propuesta por Stock-Watson (1998), obteniendo $\lambda_{g} \equiv \frac{\sigma_{5}}{\sigma_{4}}$, y cuando $z$ es no estacionario $\lambda_{z} \equiv\left(\frac{\sigma_{3}}{\sigma_{1}}\right) \frac{a_{r}}{\sqrt{2}}$. En el modelo, las ecuaciones (4) y (5) constituyen las ecuaciones de medida y las ecuaciones (2), (6) y (7) corresponden a las ecuaciones de transición dentro de un modelo básico del espacio de estados.

\subsection{Enfoque del Filtro de Kalman multivariado para estimar la tasa neutral}

La metodología del filtro de Kalman multivariado permite superar las deficiencias de los métodos econométricos que asumen parámetros que no cambian en el tiempo, como es el caso de la tasa neutral. Este método maneja el problema de la extracción de las señales de las variables de interés como un sistema de ecuaciones dinámico en el que se estima la tasa neutral $r_{t}^{*}$ y otros parámetros de manera simultánea mediante el método de máxima verosimilitud. La ventaja de este método es que la señal filtrada se da en el contexto de un modelo que incorpora la teoría económica para la identificación de los componentes de ciclo y tendencia de la dinámica de las series. Así, para estimar la tasa neutral de México, se aplica el filtro de Kalman en el sistema de ecuaciones de la (1) a la (7) enunciadas previamente. Las ecuaciones de transición en este modelo son la (2), (3), (6), y (7) y las ecuaciones de medida son la (1) y la (5). El método de estimación de los parámetros es secuencial y esta descrito de manera detallada en Laubach y Williams (2003). Primero, se aplica el filtro de Kalman, tal como lo hace Kuttner (1994), para estimar la tasa natural de crecimiento del producto, omitiendo la brecha de la tasa de interés de la ecuación (4) y asumiendo que la tasa de crecimiento

\footnotetext{
${ }^{4}$ Siguiendo la metodología de Laubach y Williams (2003), utilizamos el índice de precios de las importaciones de aceites del petróleo y aceites de minerales bituminosos, crudo, de la Reserva Federal de San Luis para incorporar el traspaso de la inflación de materias primas como el petróleo a la inflación de México.
} 
tendencial del producto g es constante. A partir de esta estimación preliminar de la tasa natural de producto se calcula la mediana de $\lambda_{g}$. Si el componente no observable de la tasa de interés neutral, z, es una caminata aleatoria, entonces se impone el valor estimado de $\lambda_{g}$ desde el primer paso y se omite la brecha de la tasa de interés real en la ecuación de la brecha del producto bajo el supuesto de que z es constante. Entonces, se estiman las otras cinco ecuaciones modelo y se obtiene una estimación de $\lambda_{z}$. En el paso final, se imponen los valores estimados de $\lambda_{g}$ del primer paso y $\lambda_{z}$ del segundo paso (bajo el supuesto de que z sigue una caminata aleatoria; de lo contrario, se estima $\sigma_{3}$ directamente) y se estiman los parámetros restantes del modelo usando el método de máxima verosimilitud, tal y como lo describe Harvey (1989). Se calculan los errores estándar correspondientes para las estimaciones de los estados usando el procedimiento de Monte Carlo de Hamilton (1986).

\subsection{Enfoque de Vectores Autor Regresivos Cointegrado (CVAR) para estimar la tasa neutral de interés.}

La metodología econométrica CVAR consiste en estimar un modelo VAR cointegrado y después imponer las restricciones que permiten para identificar la regla de Taylor en el espacio de cointegración del modelo CVAR. La estimación de la regla de Taylor, en presencia de variables con raíces unitarias, se realiza en tres pasos: 1) Se estima un modelo de Vectores Autorregresivos (VAR) que contenga la tasa de referencia $r_{t}$ y las variables de la regla de Taylor. Específicamente, el PIB real, el PIB potencial, la inflación y las expectativas de inflación. Se llevan a cabo las pruebas de correcta especificación del modelo, tales como: normalidad, no autocorrelacion y homocedasticidad. Una vez que se tiene un VAR correctamente especificado, se valida el orden de rezagos del VAR, el rango de cointegración y el tipo de polinomio determinístico del modelo. El modelo estimado se reparametriza como un modelo CVAR con base en la siguiente ecuación $\Gamma(L) \Delta z_{t}=\mu+\alpha \beta \prime z_{t-1}+\varepsilon_{t}$. Para hallar la asociación de largo plazo entre la tasa de interés nominal de referencia y las brechas del producto e inflación, se impone una serie de restricciones en la estructura del espacio de cointegración $\left(\alpha \beta / z_{t-1}\right)$. En primer lugar, se valida la existencia del número de relaciones de cointegración que son estadísticamente significativas y se impone como restricción en la estimación del CVAR. Posteriormente, en el vector de cointegración que se asocia con la regla de Taylor, se le imponen las restricciones asociadas con la ecuación $e_{t-1}=r_{t}^{*}+\beta_{1}\left(y-y^{*}\right)_{t-1}+\beta_{2}\left(\pi-\pi^{*}\right)_{t-1}$, donde $\left(y-y^{*}\right)_{t-1}$ es la brecha del producto, $\left(\pi-\pi^{*}\right)_{t-1}$ corresponde a la brecha de inflación, $e_{t-1}$ es el mecanismo de corrección de error que representa a la tasa de política monetaria y $r_{t}^{*}$ es la tasa de interés nominal neutral de largo plazo que no es observable. Para obtener estimaciones confiables es necesario que la ecuación de $e_{t-1}$ se encuentre sobre identificada en el CVAR. Así mismo, como ya hemos mencionado, se requiere validar los supuestos del modelo para confirmar la validez de las restricciones impuestas en el modelo cointegrado.

La estimación de la tasa de interés nominal neutral se obtiene restando los valores de la tasa de interés de referencia $e_{t-1}$ y los valores pronosticados por el CVAR como se indica en la siguiente expresión: $r_{t-1}^{*}=$ $e_{t-1}-\hat{\beta}_{1}\left(y-y^{*}\right)_{t-1}-\hat{\beta}_{2}\left(\pi-\pi^{*}\right)_{t-1}$. Es decir, se extrae la señal de la tasa neutral no observable $\left(r_{t}^{*}\right)$ mediante el uso de la ecuación de la regla de Taylor identificada y estimada mediante el modelo CVAR.

\section{Resultados y discusión}

Para el análisis econométrico usamos datos trimestrales del PIB real, la tasa de interés objetivo de Banxico, la inflación, las expectativas de inflación para los próximos 12 meses, el índice general de los precios de las importaciones de Banxico y los precios del petróleo (West Texas Intermediate). Estas variables se utilizan para estimar la tasa neutral mediante el modelo multivariado propuesto por Laubach y Williams (2003), que 
se sintetizó en la segunda sección de este artículo. Nuestro periodo de estimación abarca desde 2008 hasta el primer trimestre de 2020, ya que a partir de 2008 Banxico utiliza como instrumento de política monetaria la tasa de interés interbancaria a un día (Banxico, 2007). Así mismo, se obtienen las proyecciones de la tasa de interés neutral que son consistentes con el nivel de PIB potencial y una inflación estable para el periodo 2020-2024.

Para estimar el modelo, sistema de ecuaciones (1)-(7), se procede de manera secuencial como se describe en la sección de metodología. El cuadro 1 muestra los valores de los parámetros estimados que presentan el signo esperado, según el modelo teórico que comprende una curva IS para el producto y una curva de Phillips para la inflación ecuaciones (4) y (5) respectivamente.

Cuadro 1. Parámetros estimados para el modelo de Laubach y Williams (2003).

\begin{tabular}{|c|c|c|c|c|c|c|c|c|c|}
\hline Parámetro & $\lambda_{g}$ & $\lambda_{z}$ & $\sum a_{y}$ & $a_{r}$ & $b_{y}$ & $c$ & $\sigma_{1}(\hat{y})$ & $\sigma_{2}(\pi)$ & $\sigma_{3}(z)$ \\
\hline $\mathrm{Z} \sim \mathrm{AR}(2)$ & 0.052 & 0.068 & 0.428 & $-0.27(2.3)$ & $.037(1.9)$ & $0.30(7.4)$ & 0.245 & 0.617 & 0.203 \\
\hline \multicolumn{10}{|c|}{ Log likelihood } \\
\hline Parámetro & $\sigma_{4}(y *)$ & $\sigma_{s}(g)$ & $\sigma_{r} *$ & \multicolumn{5}{|c|}{-145} \\
\cline { 1 - 3 } $\mathrm{Z} \sim \mathrm{AR}(2)$ & 0.633 & 0.11 & 0.203 & & \multicolumn{5}{|c|}{ Nota: Estadístico t entre paréntesis }
\end{tabular}

Fuente: elaboración propia con base en las estimaciones del Filtro de Kalman multivariado

Una vez obtenidos los parámetros relevantes, como subproducto podemos estimar la tasa neutral que se observa en la figura 3 basada en el modelo del espacio de los estados. Estos resultados sugieren que siguiendo la tendencia a la baja, congruente con los resultados de Carrillo et al. (2018), la tasa de interés nominal neutral se encuentra por abajo del valor de la tasa de política monetaria durante casi todo el período, con excepción del 2014 y 2015.

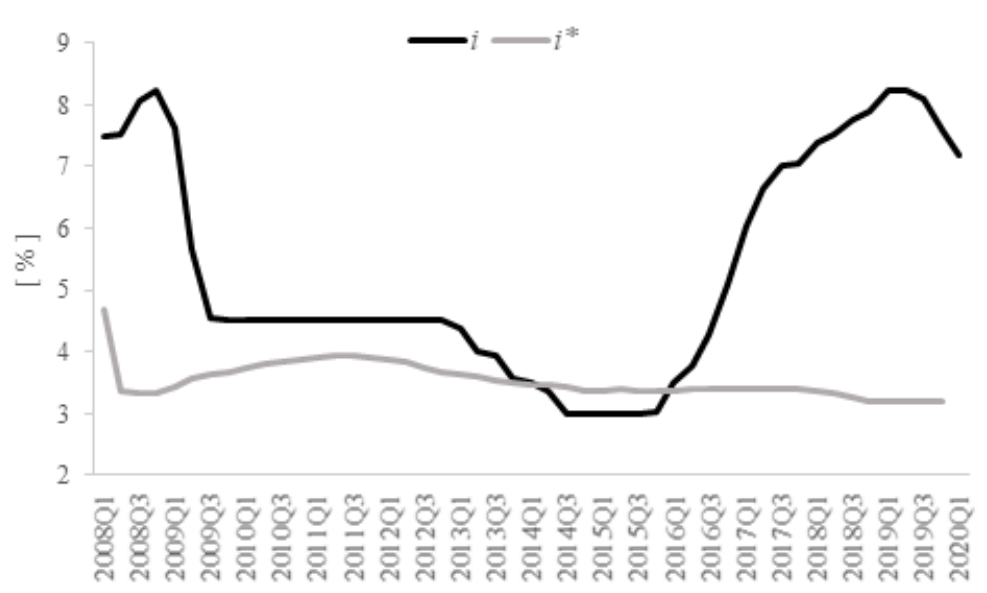

Figura 3. Tasa de interés nominal (i) y tasa de interés neutral nominal (i*)

Fuente: Elaboración propia con base en las estimaciones del Filtro de Kalman multivariado 


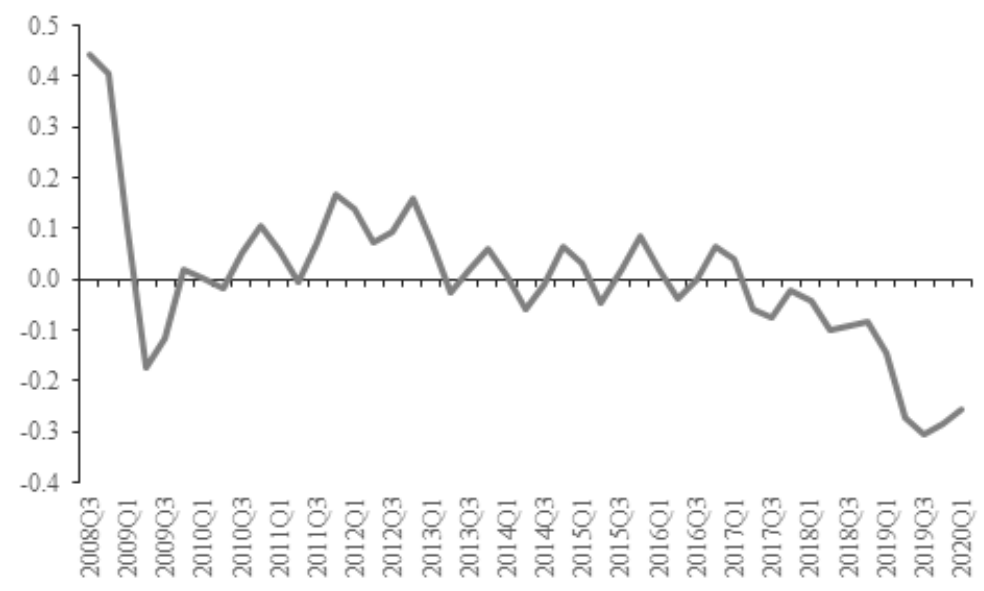

Figura 4. Brecha $\operatorname{PIB}\left(\widetilde{y}_{t}\right)$ - PIB potencial $\left(y_{t}^{*}\right)$ en porcentaje

Fuente: Elaboración propia con base en las estimaciones del Filtro de Kalman multivariado

También como un subproducto del filtro aplicado, se obtienen las estimaciones de la brecha entre el producto y el producto potencial que se muestran en la figura 4. Después de caer de un nivel de producto superior al potencial, entre 2009 y el segundo trimestre de 2017 se observa que el producto fluctúa en torno al nivel potencial o máximo que puede lograrse sin presiones inflacionarias, sin embargo, a partir de ese periodo mantiene una declinación sostenida. Esta caída continua es congruente con una tasa de interés neutral cada vez más baja.

Ahora bien, con la finalidad de verificar la robustez estadística de los resultados basados en la metodología espacio de los estados, estimamos la tasa neutral identificando la regla de Taylor en el contexto de un modelo CVAR como se sugiere en Sánchez (2016). Se estima el modelo con datos trimestrales de la tasa de interés interbancaria a un día nominal, el PIB real de México, el PIB potencial real mexicano estimado mediante el filtro de Hodrick-Prescott, la inflación y las expectativas de inflación de la encuesta sobre las expectativas de los especialistas en economía del sector privado de Banxico para el periodo 2008-2019. El modelo VAR que se reparameteriza como un CVAR tiene una constante no restringida y dos rezagos. El cuadro 2 muestra las pruebas de raíces unitarias y el cuadro 3 las pruebas conjuntas de correcta especificación de este modelo multivariado.

Cuadro 2. Pruebas de raíces unitarias (ADF Unit Root Test)

\begin{tabular}{|c|c|c|c|}
\hline $\begin{array}{c}\text { Variable } \\
\text { (misma nomenclatura) }\end{array}$ & \multicolumn{3}{|c|}{ Modelo } \\
Intercepto & Tendencia e Intercepto & Ninguno \\
\hline $\mathrm{r}$ & -1.746 & -1.672 & -0.339 \\
\hline $\mathrm{L}(\mathrm{y})$ & -0.795 & -3.435 & 3.822 \\
\hline $\mathrm{L}\left(\mathrm{y}^{*}\right)$ & 0.490 & -3.883 & 1.961 \\
\hline$\pi$ & -1.395 & -2.300 & -1.364 \\
\hline$\pi^{e}$ & -14.557 & -11.089 & -7.807 \\
\hline$\Delta r$ & -2.789 & -3.409 & -2.818 \\
\hline$\Delta L(y)$ & -4.385 & -4.372 & -3.520 \\
\hline$\Delta L(y *)$ & -2.124 & -1.602 & -1.195 \\
\hline$\Delta \pi$ & -5.298 & -5.194 & -5.323 \\
\hline$\Delta \pi^{e}$ & -4.607 & -5.170 & -4.578 \\
\hline
\end{tabular}

Nota: $\Delta$ es la primera diferencia de las series. Nivel de significancia al $5 \%$, L es el logaritmo, 
Cuadro 3. Pruebas de correcta especificación del modelo CVAR

\begin{tabular}{|c|c|c|}
\hline Prueba & Estadistico & Prob. \\
\hline Autocorrelación(F-test) & 37.11 & 0.056 \\
\hline Normalidad(JB) & 6.478 & 0.773 \\
\hline Homocedasticidad (F-test) & 291.843 & 0.377 \\
\hline
\end{tabular}

El cuadro 4 ofrece evidencia de cointegracion con base en la prueba secuencial de Johansen para la determinación conjunta del rango de cointegración (prueba de la traza) y la tendencia polinomial (Johansen 1995). Este cuadro reporta la prueba de la traza, considerando ahora la existencia de componentes deterministas en el espacio de cointegracion; el resultado de esta prueba revela que es probable que existan al menos dos vectores de cointegración al $95 \%$ de confianza.

Este procedimiento de máxima verosimilitud de Johansen permite también estimar los valores de los parámetros del espacio de cointegración. Se asume la existencia de dos vectores de cointegración y se caracteriza uno de los vectores como la ecuación de largo plazo de la tasa de interés nominal de política monetaria. Se prueba la hipótesis de que no es posible rechazar la validez estadística de las restricciones de la regla de Taylor en una de las ecuaciones del espacio de cointegración.

Cuadro 4. Prueba secuencial de Johansen (1995) de la determinación conjunta del rango de cointegración y el polinómio determinista

\begin{tabular}{|c|c|c|c|}
\hline Tipo de modelo & $\mathbf{R}$ & Traza & p-value \\
\hline I(0) Intercepto, I(1) Ninguno & 0 & 210.49 & 52.30 \\
\hline I(0) Intercepto, I(1) Intercepto & 0 & 358.10 & 0.00 \\
\hline I(0) Intercepto, I(1) Ninguno & 1 & 101.59 & 33.60 \\
\hline I(0) Intercepto, I(1) Intercepto & 1 & 137.49 & 0.00 \\
\hline I(0) Intercepto, I(1) Ninguno & 2 & 36.19 & 18.70 \\
\hline I(0) Intercepto, I(1) Intercepto & 2 & 43.02 & 0.00 \\
\hline I(0) Intercepto, I(1) Ninguno & 3 & 17.02 & 7.55 \\
\hline I(0) Intercepto, I(1) Intercepto & 3 & 23.14 & 0.01 \\
\hline I(0) Intercepto, I(1) Ninguno & 4 & 3.32 & 0.44 \\
\hline I(0) Intercepto, I(1) Intercepto & 4 & 8.36 & 0.07 \\
\hline
\end{tabular}

Nota: $\mathrm{R}=$ rango de cointegración; Ninguno=se refiere a que el tipo de modelo estimado no tiene ni tendencia ni constante.

Fuente: Elaboración propia con base en el modelo CVAR.

Cuadro 5. Vector de cointegración normalizado como una ecuación de la tasa de interés interbancaria a un

$$
\begin{gathered}
\text { día } \\
r_{t-1}=r_{t-1}^{*}-0,459\left(y-y^{*}\right)_{t-1}-1,175\left(\pi-\pi^{*}\right)_{t-1}
\end{gathered}
$$

Prueba de sobre identificación LR-CHISQR $(2)=8.229070[0.016334]$

Fuente: Elaboración propia con base en el modelo CVAR estimado

El cuadro 5 muestra que las restricciones que permiten identificar la regla de Taylor en el espacio de cointegracion del CVAR son estadísticamente apropiadas. Se concluye que los datos de la economía mexicana aceptan las restricciones correspondientes a la regla de Taylor con un nivel de confianza del 95 por ciento. En el cuadro se muestra el vector de cointegración normalizado como una ecuación de la tasa de interés de fondeo a un día. Esta relación de largo plazo indica que la tasa de interés de política económica aumenta ante desviaciones en las brechas de producto e inflación, tal como se menciona en el modelo teórico, Taylor (1993) y Clarida (2015). Esta estimación sugiere que la tasa de política monetaria aumenta en mayor grado cuando la brecha de inflación se amplia, lo que está en línea con el mandato único del banco central. 
Cuadro 6. Tasa de interés neutral real y nominal $\left(r_{t}^{*}\right)$ con los diferentes métodos

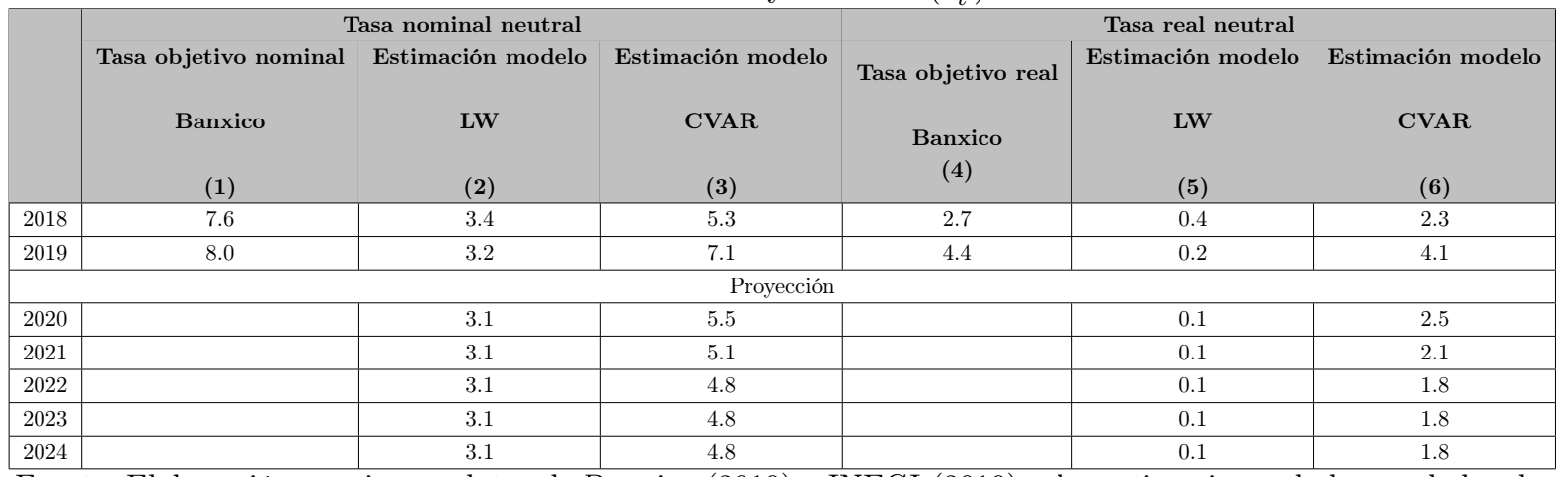

Fuente: Elaboración propia con datos de Banxico (2019) e INEGI (2019) y las estimaciones de los mode-los de

$$
\text { Laubach y Williams (LW) y CVAR }
$$

El cuadro 6 muestra las proyecciones, con las metodologías de Laubach y Williams (2003) y del modelo CVAR, de la tasa de interés nominal neutral y la tasa de interés real neutral ${ }^{5}$ para México durante el periodo 2019-2024. Estas proyecciones sugieren que, dados los pronósticos de la brecha de producto y de la brecha de inflación, tanto la tasa de interés nominal neutral como la tasa de interés real neutral se encuentran por abajo del valor de la tasa de política monetaria que fijó el Banco de México en los últimos meses de 2019 y principios de 2020. Lo anterior implica que la postura actual de política monetaria del Banco de México es actualmente más restrictiva que acomodaticia. En consecuencia, la tasa de referencia pudiera recortarse al menos 50 o 100 puntos base adicionales para estimular la actividad económica.

Las estimaciones con el modelo del espacio de los estados y el CVAR nos permiten discutir la relevancia que podría jugar la tasa de referencia como una política anti-cíclica a partir de la crisis del Covid-19. Observamos que las estimaciones del modelo de Laubach y Williams (2003), LW, sugieren que durante 2018 y 2019 Banxico implementó una tasa de interés objetivo por arriba de la tasa neutral (ver columnas 2 y 5 ); lo mismo se confirma con el modelo CVAR (columnas 3 y 6 ).

Específicamente, las estimaciones del modelo de Laubach y Williams (2003) sugieren que durante 2018 y 2019, México tuvo tasas reales neutrales de alrededor de $0.4 \%$ y $0.2 \%$ respectivamente. Por lo tanto, si consideramos una inflación promedio de alrededor del $3 \%$ obtenemos tasas neutrales nominales de $3.4 \%$ y $3.2 \%$ para esos dos años. Las tasas objetivo de Banxico fueron de $7.6 \%$ y $8 \%$, lo que implica que la postura de política monetaria fue efectivamente muy contractiva en estos dos años según nuestros cálculos.

Por otra parte, si consideramos las estimaciones con el modelo CVAR encontramos que en 2018 y 2019 las tasas reales neutrales debían fluctuar en torno a $2.3 \%$ y $4.1 \%$. En consecuencia, las tasas neutrales nominales deberían ser de alrededor de $5.3 \%$ y $7.1 \%$ en 2018 y 2019. Estos valores, de cualquier manera, son menores que los objetivos implementados por Banxico en ese período. En consecuencia, ambos modelos sugieren que la postura del banco fue contractiva. El banco central aún tenía espacio para reducir su tasa de interés e implementar una política monetaria más expansiva sin generar presiones inflacionarias en estos dos años. Sin embargo, privilegiando su mandato de mantener una inflación controlada Banxico decidió mantener una tasa de interés mucho más alta que la que sugieren las estimaciones de la tasa neutral.

En abril de 2020 el Banco de México decidió bajar la tasa de referencia a $6 \%$ ante la disminución de la actividad económica en 2019, por primera vez en casi 2 años de mantenerla en un rango de 7 a $7.5 \%$; fijándola en $5 \%$ en junio. Nuestros resultados confirman que actualmente existe una recesión económica y que Banxico aún tiene espacio para un periodo más largo de política monetaria expansiva en México. La tasa real neutral estimada con el modelo de Laubach y Williams (2003) se reduce a un nivel de aproximadamente 0.1 puntos

\footnotetext{
${ }^{5}$ La tasa de interés real neutral se obtiene restando las expectativas de inflación a la tasa neutral nominal proyectada por el CVAR.
} 
base en el periodo 2020-2024 y se mantiene constante hasta 2024, mientras que la tasa nominal neutral es de alrededor de $3.1 \%$ en el mismo período.

Los resultados del modelo de Laubach y Williams (2003) son consistentes en la dirección de la tasa neutral real de interés estimada con el modelo CVAR. Este último sugiere que la tasa real neutral debería disminuir hasta $2.5 \%$ en 2020 y llegar hasta $1.8 \%$ en 2024 . Esto implica que la tasa nominal neutral debe iniciar en $6 \%$ en 2020, alcanzar un $5.1 \%$ en 2021 y mantenerse en $4.8 \%$ durante el periodo 2022-2024. Es decir, todavía es posible reducir aún más la tasa de interés de política monetaria con la finalidad de contribuir a llevar a cabo una política contra-cíclica. Cabe destacar que el valor de $4.8 \%$ se acerca al valor de la tasa de interés de $4.5 \%$ que tuvo lugar en el periodo 2009-2012 posterior a la crisis de 2008 como puede observarse en la figura 2 .

Aún más, el modelo de Laubach y Williams (2003) sugiere que es posible llevar aún más allá la postura expansiva del Banco Central hasta alcanzar una tasa de política monetaria cercana a $3 \%$, ya que la tasa neutral observaría un valor de $0.1 \%$ en términos reales y a $3.1 \%$ en términos nominales. Un argumento más a favor de una política monetaria un poco más expansiva está dado por el hecho de que en este año es posible que la caída del producto exceda de $5 \%$ y es también posible que la inflación se mantenga en niveles bajos comparables con lo sucedido en la crisis de 2008. Tanto la evidencia histórica, como nuestras cifras de prospectiva, sugieren que el banco central debería contribuir disminuyendo la tasa de interés al menos hasta un $4.5 \%$.

La posición actual de una tasa de interés de $6 \%$ no es apropiada dados los posibles cambios en la demanda agregada, las preferencias de los consumidores y el PIB potencial. Dichos cambios vendrán a partir de la crisis y serán los nuevos fundamentos a partir de 2020. Si Banxico determina reducir la tasa a partir de 2020, se generarían ganancias económicas en México. Según nuestras proyecciones, la tasa real neutral seguirá siendo muy baja hasta 2021, lo que significa que la economía continuará experimentando una inflación muy por debajo del objetivo de $3 \%$.

Por otra parte, dado que la tasa nominal neutral es positiva pero muy baja, entonces se indica la necesidad de que el estímulo monetario no se reduzca agresivamente en el periodo que comprende 2021-2024. Una postura contractiva podría resultar en una contracción con baja inflación. La razón de este resultado es que el PIB probablemente sea más bajo que el PIB potencial y el crecimiento del crédito también será muy bajo.

\section{Conclusiones}

Encontramos evidencia de que Banxico aún tiene margen para reducir su tasa de interés objetivo sin generar problemas inflacionarios. Lo anterior es resultado del choque en la oferta y la demanda, derivados de la crisis sanitaria y económica del Covid-19 por la necesidad de que se tomen medidas de contención de la propagación de la enfermedad que afectan a las cadenas productivas y al consumo de las personas. En general, parece que México requiere de una intervención masiva de política monetaria para impulsar la economía como se hizo en 2008, véase Banxico (2008, pp. 83-84) y durante 2009. El estímulo monetario debería continuar durante al menos tres años para que la economía reanude el crecimiento sostenido con una inflación dentro de la meta. Demasiada anticipación en aumentar la tasa de interés podría resultar en una mayor contracción en México.

Es importante mencionar que debido a que la tasa de interés neutral es una variable no observable y se emplean diferentes métodos para aproximar su valor, nuestros resultados son sugerentes y pueden servir como indicativos para evaluar la posición de política monetaria del Banco de México durante la crisis del Covid-19. Nuestro modelo proyecta una tasa de interés real neutral muy baja hasta el último trimestre de 2020 (que es sustancialmente más baja que la tasa de interés real del banco actualmente). La economía de 
México seguirá experimentando choques de oferta y demanda que cambiarán de manera continua no sólo las preferencias de los consumidores, sino también el PIB potencial, lo que modificará la tasa neutral a la baja y, en consecuencia, la tasa de interés de referencia debería reacomodarse de manera constante.

\section{Agradecimientos}

El artículo es resultado conjunto del Programa de Apoyo a Proyectos de Investigación e Innovación Tecnológica, Proyecto PAPIIT IN302419, y del Programa de Apoyo a Proyectos para Innovar y Mejorar la Educación, Proyecto PAPIME PE310919, ambos de la Dirección General de Asuntos del Personal Académico (DGAPA) de la UNAM. Los autores agradecen a Gonzalo Curiel Vázquez, Lizeth Guerrero González, Débora Martínez Ventura, Raúl Orozco y Ruth Martínez por su asistencia técnica.

\section{Referencias}

[1] Banxico (2007). Instrumentación de la Política Monetaria a través de un Objetivo Operacional de Tasa de Interés. (Anexo 3 del informe sobre Inflación Julio-Septiembre 2007), Banxico. https://www.banxico.org.mx/politicamonetaria/d/

[2] Banxico (2008). Informe sobre la Inflación julio-septiembre 2008. (Informe sobre Inflación julio-septiembre 2008), Banxico. https://www.banxico.org.mx/publi caciones-y-prensa/informes-trimestrales/

[3] Banxico (2019). Sistema de Información Económica. https://www.banxico.org.mx/SieIn ternet/.

[4] Barro, R. J., Ursúa, J. F. y Weng J. (2020). The Coronavirus and the Great Influenza Pandemic: Lessons from the "Spanish Flu"for the Coronavirus's Potential Effects on Mortality and Economic Activity. NBER Working Paper Series, National Bureau of Economic Research, Working Paper 26866. https://www. nber.org/papers/w26866.pdf

[5] Behera, H. K., Pattanaik, S. y Kavediya, R. (2017). Natural interest rate: Assessing the stance of Indias monetary policy under uncertainty, Journal of Policy Modeling, 39(3), 482-498. https://doi.org/10.1016/j.jpolmod.2017.03.002

[6] Bell, C. y Lewis, M. (2004). The economic implications of epidemics old and new. World Economics, 5(4), 137-174. http: //eds. b.ebscohost.com. pbidi.unam.mx: 8080/eds/pdfviewer/pdfviewer?vid=2sid=cb7f55d434c6-462f-9141-b6a50239 da 3d

[7] Carrillo, J., Elizondo, R., Rodríguez-Pérez, C. A. y Roldán-Peña, J. (2018). What Determines the Neutral Rate of Interest in an Emerging Economy? Banco de Mexico. Working paper N 2018-22. https://www.banxico.org.mx/publications-and-press/banco-de-mexico-working-papers/

[8] Cerra, V. y Saxena, S. C. (2017). Booms, Crises, and Recoveries: A New Paradigm of the Business Cycle and Its Policy Implications, IMF Working Papers, WP/17/250. https://www.imf.org/en/Publications/WP/Issues/2017/11/16/Booms-Crises-and-Recoveries-A-New-Paradigmof-the-Business-Cycle-and-its-Policy-Implications-45368

[9] Clarida, R., (2015). The Fed is ready to raise rates: Will past be prologue? International Finance, 18(1), 1-15. https://doi.org/10.1111/1468-2362.12059

[10] Cochrane, J. H. (2020). Strategic Review and Beyond: Rethinking Monetary Policy and Independence. Economic Research Federal Reserve Bank of St. Louis, Second Quarter 2020, 102(2), 99-119. https://doi.org/10.20955/r.102.99-119

[11] Dietrich, A. M., Kuester, K., Müller, G. J. y Schoenle, R. S. (2020). News and Uncertainty about COVID19: Survey Evidence and Short-Run Economic Impact. Federal Reserve Bank of Cleveland. Working paper 20-12. https://www. clevelandfed.org/en/newsroom-and-events/publications/working-papers/2020-workingpapers/wp-2012-consumer-expectations-about-covid19-impact.aspx

[12] Eichenbaum, M. S., Rebelo, S. y Trabandt, M. (2020). The macroeconomics of epidemics. National Bureau of Economic Research. Working Paper 26882. https ://www.nber.org/papers/w26882.pdf 
[13] España-Arias, V. (2008). La Tasa Natural de Interés: Estimación para la Economía Uruguaya. Revista de Economía. 15(2), 23-66. http://www.bvrie.gub.uy/local/Fi le/REVECO/2008/Espania.pdf

[14] Fuentes, R y Gredig F. (2008) La Tasa de Interés Neutral: Estimaciones para Chile. Economía Chilena. 11(2), 47-58. https://repositoriodigital.bcentral.cl/xmlui/ bits tream/handle/20.500.12580/3505/BCCh-recv11n2ago2008p047058.pdf? sequen ce $=1$ isAllowed $=y$

[15] Furman, J. (2020). Protecting People Now, Helping the Economy Rebound Later en R. Baldwin y B. Weder di Mauro Editors, Mitigating the COVID Economic Crisis: Act Fast and Do Whatever It Takes, (pp. 191-96) Center for Economic Policy and Research. Washington, D.C.: CEPR Press. https://voxeu.org/content/mitiga ting-covid-economic-crisis-act-fast-and-do-whatever-it-takes

[16] Galí, J. (2020). Helicopter Money: The Time Is Now en R. Baldwin y B. Weder di Mauro Editors, Mitigating the COVID Economic Crisis: Act Fast and Do Whatever It Takes (pp. 57-62). Center for Economic Policy and Research. Washington, D.C.: CEPR Press. https://voxeu.org/content/mitigating-covid-eco nomic-crisis-act-fastand-do-whatever-it-takes

[17] Gopinath, G. (2020). Limiting the Economic Fallout of the Coronavirus with Large Targeted Policies en R. Baldwin y B. Weder di Mauro Editors, Mitigating the COVID Economic Crisis: Act Fast and Do Whatever It Takes (pp. 41-48). Center for Economic Policy and Research. Washington, D.C.: CEPR Press. https://voxeu.org/content/mitigating-covid-economic-crisis-act-fast-and-do-what ever-it-takes

[18] Gourinchas, P-O. (2020). Flattening the pandemic and recession curves. mimeo UC Berkeley. https://clausen. berkeley.edu/wp-content/uploads/2020/03/COVID_2b.pdf

[19] Goyal, A. y Arora, S. (2016). Estimating the Indian natural interest rate: A semi-structural approach. Economic Modelling, 58, 141-153. https://doi.org/10.1016/j .econmod.2016.05.023

[20] Hamilton, J. (1986). A standar error for the estimated state vector of a state-space model. Journal of Economics, 33(3), 387-397. https://doi.org/10.1016/0304-407 6(86)90004-7

[21] Harvey, A. (1989). Forecasting, structural time series models and the kalman filter. Cambridge University Press.

[22] Hassan, T. A., Hollander, S., van Lent, L. y Tahoun, A. (2020). Firm-Level Exposure to Epidemic Diseases: Covid-19, SARS, and H1N1. Institute for New Economic Thinking, Working Paper No. 119. https://www.nber.org/papers/w26971.pdf

[23] Holston, K., Laubach, T. y Williams, J. C. (2017). Measuring the Natural Rate of Interest: International Trends and Determinants. Journal of International Econo-mics, 108(1), 59-75. https://doi.org/10.1016/j.jinteco.2017.01.004

[24] INEGI (2019). Banco de Información Económica. https://www.inegi.org.mx/sistemas/ bie/

[25] Kalman, R. E. (1960). A New Approach to Linear Filtering and Prediction Problems, Transactions of the ASME - Journal of Basic Engineering, 82, 35-45. http://ww w.unitedthc.com/DSP/Kalman1960.pdf

[26] Krugman, P. (2020). The case for permanent stimulus en R. Baldwin y B. Weder di Mauro Editors, Mitigating the COVID Economic Crisis: Act Fast and Do Whatever It Takes (pp. 213-219). Center for Economic Policy and Research. Washington, D.C.: CEPR Press. https://voxeu.org/content/mitigating-covid-eco nomic-crisis-actfast-and-do-whatever-it-takes

[27] Kuttner, K. (1994). Estimating potencial output as a latent variable. Journal of Business Economic Stadistics, 12(3), 361-368. DOI: $10.1080 / 07350015.1994 .10524551$

[28] Laubach, T. y Williams, J. (2001). Measuring the Natural Rate of Interest. Board of Governors of the Federal Reserve System (US). https://www.federalreserve.gov/ pubs/feds/2001/200156/200156pap.pdf

[29] Laubach, T. y Williams, J. C. (2003). Measuring the natural rate of interest. Review of Economics and Statistics, 85(4), 1063-1070. https://www.jstor.org/stable/321182 6

[30] Laubach, T. y Williams, J. C. (2015). Measuring the Natural Rate of Interest Redux. Federal Reserve Bank of San Francisco, Working Paper 2015-16. http://www. frbsf.org/economic-research/publications/workingpapers/wp2015-16.pdf 
[31] Méssonnier, J. S. y Renne, J. P. (2007). A time varying "natural" rate of interest for the euro area. European Economic Review, 51(7), 1768-1784. https://doi.org/10.101 6/j.euroecorev.2006.11.006

[32] Nicola, M., Alsafi, Z., Sohrabi, C., Kerwan, A., Al-Jabir, A., Iosifidis, Ch., Aghae, M. y Aghaf, R. (2020). The socio-economic implications of the coronavirus pandemic (COVID-19): A review. International Journal of Surgery, 78, 185-193. https:// doi.org/10.1016/j.ijsu.2020.04.018

[33] Odendahl, C., y Springford, J. (2020). Bold Policies Needed to Counter the Coronavirus Recession en R. Baldwin y B. Weder di Mauro Editors, Mitigating the COVID Economic Crisis: Act Fast and Do Whatever It Takes (pp. 145-150). Center for Economic Policy and Research. Washington, D.C.: CEPR Press. https://voxeu. org/content/mitigating-covid-economic-crisis-act-fast-and-do-whatever-it-takes

[34] Ozili, P. K. y Arun, T. (30 de abril de 2020). Spillover of COVID-19: Impact on the Global Economy. http://dx.doi.org/10.2139/ssrn.3562570

[35] Ramsey, F. P. (1928) A mathematical theory of saving. The Economic Journal, 38(152), 543-559. DOI: $10.2307 / 2224098$

[36] Sánchez, A. (2016). Should the U.S. Federal Reserve increase the federal funds rate in 2016? An assessment based on the neutral interest rate. Investigación Económica. 75(296), 5-42. https://doi.org/10.1016/j.inveco.2016.07.002

[37] Schmitt-Grohé, S. y Uribe M. (2018). How important are terms of trade shocks? International Economic Review, 59(1), 85-111. https://doi.org/10.1111/iere.122 63

[38] Taylor, J. B. (1993). Discretion versus Policy Rules in Practice, Carnegie Rochester Conference Series on Public Policy, 39, 195-214. https://doi.org/10.1016/0167-2231(93)90009-L

[39] Zhang, D., Hua, M. y Ji, Q. (2020). Financial markets under the global pandemic of COVID-19. Finance Research Letters, en prensa. https://doi.org/10.1016/j.frl. 2020.101528 